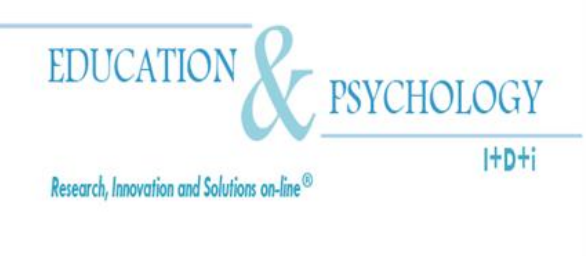

\title{
Influencia de los factores familiares en el abandono escolar temprano. Estudio de un contexto multicultural
}

\section{Gloria Rojas Ruiz ${ }^{1}$, Inmaculada Alemany Arrebola ${ }^{2}$, $M^{a}$ Mar Ortiz Gómez ${ }^{2}$}

${ }^{1}$ Departamento de Didáctica y Organización Escolar, Universidad de Granada, Campus Universitario de Melilla.

${ }^{2}$ Departamento de Psicología Evolutiva y de la Educación, Universidad de Granada, Campus Universitario de Melilla.

\section{España}




\section{Resumen}

Introducción. En las investigaciones sobre abandono escolar temprano encontramos una gran variedad de factores que influyen en este problema. Los informes más actuales señalan como más relevantes en los resultados escolares del alumnado, el entorno educativo y el ambiente familiar y social.

Método. Los objetivos de este artículo se centran en conocer el nivel socioeconómico y cultural de los padres de alumnos que abandonan prematuramente los estudios y evaluar la implicación familiar en el progreso educativo de los hijos, en un contexto multicultural. La metodología utilizada es descriptiva. Se evaluó a través de una encuesta a jóvenes de entre 16 y 24 años que abandonaron prematuramente los estudios desde el año 2000-2001 hasta el 20082009.

Resultados. Los resultados de este trabajo muestran que el nivel socioeconómico de las familias de los alumnos que abandonan prematuramente es bajo, encontrándose mayor porcentaje de desempleo en familias de origen bereber, frente a las de origen europeo. El nivel de estudios de los progenitores es de Educación Primaria o Educación Secundaria Obligatoria, teniendo los padres mayor formación que las madres y mayor preparación académica los de procedencia europea.

Conclusiones. Aunque los datos del Informe PISA muestran que un rendimiento pobre en la escuela no es consecuencia automática de un entorno socioeconómico desfavorecido, el ambiente familiar parece influir poderosamente en el rendimiento. Los resultados de este trabajo coinciden con numerosos estudios sobre la relación directa existente entre las circunstancias familiares y su incidencia en el abandono escolar temprano.

Palabras Clave: Abandono Escolar Temprano; Variables Socioculturales; Variables familiares; Contexto multicultural. 


\title{
Influence of family factors in school drop-out: a study within a multicultural context
}

\begin{abstract}
Introduction. Research on early school leaving shows a great variety of factors that influence this problem. The latest reports point out as more relevant to students'school performance, school environment and family and social circumstances.

Method. The objectives of this article focus on knowing the socioeconomic and cultural level of the parents of students who leave school early and evaluate the family involvement in the educational progress of children in a multicultural context. The methodology used is descriptive. It was evaluated through a survey of young people between 16 and 24 years who left school early from 2000-2001 to 2008-2009.

Results. The results of this study show that the socioeconomic status of families of students who drop out is low, existing a highest percentage of unemployment in families of Berber origin, compared to those of European origin. The educational level of parents is of primary education and compulsory secondary education, fathers have a higher education than mothers and a better academic training those from Europe.
\end{abstract}

Conclusions. Although data from PISA shows that poor performance in school is not an automatic consequence of a disadvantaged socioeconomic background, family environment seems to strongly influence performance. The results of this study are consistent with numerous studies on the relationship between family circumstances and their impact on early school leaving.

Keywords: early school leaving. Sociocultural Variables. Family variables. Multicultural context. 


\section{Introducción}

Los grandes avances educativos que se han producido en España en los últimos treinta años, incorporando la obligatoriedad y la universalidad de la enseñanza, unidos al esfuerzo de toda nuestra sociedad para favorecer la igualdad de oportunidades, ha posibilitado la participación de los ciudadanos en la vida política, económica, cultural y social del Estado, que, no obstante, se ha visto a veces disminuida por el alto grado de fracaso y abandono escolar temprano de muchos estudiantes, dificultando su futura integración social y laboral. El Consejo de la Unión Europea, en el año 2006, destacó que la reducción de esta situación puede aumentar la empleabilidad y favorecer la cohesión social y el modelo social europeo. Siguiendo en esta línea, Feito (2009) detalla que, en la actualidad, no poseer los mínimos educativos básicos obstaculiza, no sólo la posibilidad de encontrar un buen empleo, sino también el desarrollo de una ciudadanía digna.

La preocupación por este problema ha supuesto que uno de los objetivos y puntos de referencia establecidos por la Unión Europea para el año 2010, en materia de educación, fuera reducir a la mitad el número de alumnos que abandonan prematuramente el sistema educativo, comparando estos datos con los del año 2000. No obstante, en el informe Progress towards the Common European objectives in education and training (Commision of the European Communities, 2011), se refleja que el abandono escolar sigue siendo una de las tareas pendientes de nuestro continente, ya que uno de cada siete jóvenes de la Unión Europea sigue presentando esta situación.

Según los resultados de dicho informe y, a pesar de que uno de los propósitos fundamentales de la educación en nuestro país es el de alcanzar el éxito de todos los alumnos en la enseñanza obligatoria, España sigue teniendo un $31 \%$ de abandono escolar, el doble de la media europea. Este dato demuestra que nuestro sistema educativo no ha conseguido mejoras en este aspecto, puesto que ya, en el año 2007, presentaba el mismo porcentaje, frente a un 14.8\% del resto de Europa (MEPSYD, 2008, p. 32). Por todo esto y, siguiendo a López Rupérez (2010, p.43), esta situación "reclama de los poderes públicos y de la propia sociedad una reflexión profunda sobre las causas, con la ayuda de investigaciones sistemáticas, que, en nuestro país, aún son escasas". 
Cuando abordamos las investigaciones y los estudios sobre esta situación, se constata que existen múltiples definiciones que se manejan en la literatura relacionada y que resultan ambiguas (Instituto Vasco de Evaluación e Investigación Educativa - IVEI-2007), tales como el fracaso escolar, término más usado en España que en el resto de países europeos, el abandono escolar temprano o el absentismo escolar. Un informe del Fondo de Investigación de la Protección Social, FIPROS, (Albert, 2008) determina tres conceptos muy relacionados: retraso escolar, fracaso escolar y abandono escolar temprano. Este trabajo define el retraso escolar como el que tienen aquellos jóvenes que logran el Graduado en Educación Secundaria Obligatoria fuera de la edad teórica de finalización de esos estudios marcada por la LOE (16 años); el colectivo de alumnos que no consiguen tal graduación sufren fracaso escolar; y aquellos que la han alcanzado pero no continúan en el sistema educativo reglado son casos de abandono escolar temprano al no ser demandantes de estudios post-obligatorios. Roca (2010), para establecer las diferencias entre fracaso y abandono, detalla que el primer concepto se refiere a la educación obligatoria y el segundo, a la postobligatoria, aunque la relación entre ellos es inseparable, puesto que el abandono temprano está condicionado, en la mayoría de las ocasiones, por el fracaso de los que, al terminar los estudios obligatorios, no han obtenido el título de ESO. La definición más empleada para precisar el concepto de abandono escolar temprano es la acordada por el Consejo Europeo de Bruselas, en el año 2003, que se refiere al porcentaje de jóvenes de 18 a 24 años que han alcanzado como máximo la educación secundaria obligatoria y no continúan estudiando o formándose, para acceder a una inserción laboral más cualificada.

En las investigaciones sobre esta temática, encontramos una gran variedad de factores que ejercen su influencia en el fenómeno del abandono escolar temprano. Si bien, en un principio, los enfoques teóricos y las investigaciones que trataron el problema se centraron en el individuo (Lee y Burkhan, 2000), dejando a un lado los referentes sociales, políticos y organizativos, como pudiera ser la acción de la propia escuela, en estos momentos se analiza la interrelación de todos estos factores en el contexto educativo (Newman, Wehlage y Lamborno, 1992, Finn, 1993, Wilms, 2000, Rubenger, 2001 y Lehr, Johnson, Bremer, Cosio y Thompson, 2004). Como señala González (2006, p.4), “el abandono escolar no es sino la etapa final de un proceso acumulativo y dinámico de desenganche [de la escuela]".

Investigaciones e informes actuales sobre el tema señalan como influyentes los factores individuales (Marchesi, 2003, Calero, Choi y Waisgrais, 2010, Poy, 2010) del entorno educa- 
tivo (Railsback, 2004, Robledo, Cortez y Cortez, 2004, MEPSYD, 2008) y del ambiente familiar y social. Este trabajo se centra principalmente en las variables familiares, ya que cada vez está más extendida y desarrollada la idea de que el nivel educativo de la familia y la implicación en la formación de sus hijo influyen directamente en el progreso y el rendimiento académico de los alumnos (Lozano, 2003). Las características socioeconómicas y el grado de estudios alcanzado por los progenitores, sobre todo de la madre, intervienen en los resultados escolares de los hijos (Marchesi, 2003; OECD, 2004 y 2009; Pajares, 2005; Escardíbul, 2008; Casquero y Navarro, 2008); de hecho, el Informe PISA-2006 lo señala como el factor más influyente, aunque no sea determinante.

$\mathrm{Al}$ desarrollarse este estudio en un contexto multicultural, el origen cultural es otro de los aspectos que se ha tenido en cuenta, ya que, según el Informe sobre Fracaso y Abandono Escolar en España (Enguita, Mena y Riviere, 2010), algunas comunidades con gran arraigo a sus tradiciones, presentan mayor índice de abandono escolar temprano. Dicho informe refleja que esta situación está totalmente relacionada con las características familiares, ya que, muchos de los alumnos pertenecientes a minorías culturales o hijos de inmigrantes que abandonan los estudios, provienen de hogares cuyos padres están empleados en los sectores menos cualificados.

La prodecencia cultural de los grupos que han participado en nuestro trabajo corresponde al grupo bereber y al grupo de procedencia europea. En este sentido, es importante destacar que la ciudad de Melilla es una de las que peores resultados educativos obtiene a nivel nacional y con unas tasas de paro muy elevadas $(21.02 \%)$. La mayor parte de los desempleados habitan en la denominada Zona Norte, en la que predomina la población de origen bereber y el mayor número de personas de procedencia extranjera -tres de cada diez habitantes de esos distritos son de Marruecos-y donde el $41 \%$ de los hogares no cuenta con ninguno de sus miembros que trabaje en la actualidad (Segura Vázquez, 2010, p.8).

En esos barrios melillenses llama la atención el elevado porcentaje de cabezas de familia que no tienen estudios: en tres de cada cuatro hogares esta persona carece de ellos o sólo tiene primarios incompletos. En otros términos, según el estudio realizado por Segura Vázquez (p. 109), sólo en uno de cada cuatro hogares el cabeza de familia tiene, al menos, el graduado escolar, dato muy lejano de la media española considerando que el $66 \%$ de los cabeza de familia tienen por lo menos la EGB, ESO o el título de Graduado Escolar. Esto con- 
lleva sobre todo a un empleo precario, circunstancia que, en la mayoría de las investigaciones, se relaciona directamente con el abandono prematuro de los estudios de los hijos. No obstante, el MEPSYD, en su Plan para la Reducción del Abandono Escolar del año 2008, detalla que en algunas culturas, las familias con un nivel socio-económico bajo, son más ambiciosas en los logros educativos de sus hijos que las de un nivel socio-económico más alto, por lo que no parece ser un factor determinante del abandono.

\section{Objetivos}

Este artículo se enmarca dentro de una investigación más amplia que sobre el Abandono Escolar Temprano se ha realizado entre diciembre de 2008 y julio de 2010 en Melilla, como desarrollo del "Convenio de colaboración entre el MEPSYD y la Universidad de Granada para la puesta en marcha de iniciativas conjuntas dirigidas a la mejora de la calidad de la atención educativa de las personas con escasa titulación, a la reducción del abandono temprano de la escolarización y al perfeccionamiento de la formación del profesorado no universitario en las ciudades de Ceuta y Melilla”. El trabajo que se presenta está centrado en la influencia de los de los factores familiares en sujetos con abandono escolar temprano en la Ciudad Autónoma de Melilla, en el momento de dejar los estudios. Para ello se plantean los siguientes objetivos:

- Conocer el nivel socioeconómico y cultural de los padres de los alumnos que han abandonado el sistema educativo de forma prematura.

- Evaluar su implicación familiar en el progreso educativo de los hijos.

- Analizar cómo el grupo de procedencia cultural influye en el Abandono Escolar Temprano.

Las hipótesis que se plantean en esa investigación son:

- El nivel socioeconómico y cultural de los padres influyen en el Abandono Escolar Temprano.

- La implicación de las familias en la educación de sus hijos está relacionada con el Abandono Escolar Temprano.

- La procedencia cultural incide en el Abandono Escolar Temprano. 


\section{Método}

\section{Participantes}

El Ministerio de Educación, a través de este estudio, solicitó evaluar el Abandono Escolar Temprano entre los jóvenes de edades comprendidas entre los 16 y 24 años de la Ciudad Autónoma de Melilla (ME, 2010). Para ello se ha utilizado un muestreo no probabilístico de tipo causal o incidental, puesto que se ha elegido directa e intencionalmente a los sujetos que iban a participar en este estudio (Buendía, 1997), por su accesibilidad y la voluntad de colaborar. Para la selección de los participantes se partió de la base de datos suministrada por los Institutos de Educación Secundaria (IES) de la Ciudad Autónoma de Melilla, compuesta por un total de 3362 sujetos que habían abandonado sus estudios, desde el año 2000-2001 hasta el 2008-2009. Sólo se pudo contactar con 940 personas, ya que el resto era ilocalizable con los datos que se poseían. A éstos se les explicó el trabajo que se estaba realizando y se les ofreció la posibilidad de participar en el mismo; de ellos, sólo 190 sujetos accedieron, el resto alegó haber continuado sus estudios con posterioridad o simplemente que no deseaba colaborar.

La muestra definitiva está compuesta por 93 hombres y 97 mujeres. Al ser el lugar de estudio una ciudad multicultural, se ha tenido en cuenta la variable cultura de procedencia en nuestros análisis, así, de procedencia musulmana contamos con un 50\% de nuestra muestra; cristiana, un $47.9 \%$,; y de otras, en un $2.2 \%$. Por otra parte, el rango de edad de los participantes oscila entre los 16 y 24 años, con una edad media de 21.09 y una desviación típica de 2.352 .

\section{Instrumentos}

Para recopilar la información se ha elaborado un cuestionario ad hoc. Los ítems, en su mayoría, hacen referencia al período en el cual el joven dejó de ir al instituto, a excepción de algunas preguntas referidas a su situación actual. El proceso seguido en la construcción del Cuestionario de Abandono Escolar Temprano (AET) pasó por distintas etapas hasta lograr el cuestionario definitivo (ME, 2010). En este instrumento se han considerado cinco dimensiones o bloques (datos personales y familiares, aspectos escolares, aspectos familiares y sociales, el abandono escolar y la iniciación laboral) que agrupan o clasifican a un total de 114 ítems. Para obtener la validez de contenido del cuestionario final, se realizó tras la aplicación 
de la técnica de juicio de expertos, a través de la cual, los 7 expertos consultados (docentes, orientadores de IES de la Ciudad de Melilla y expertos en metodología de investigación educativa) debían valorar, en una escala de 1 a 5, cada uno de los ítem en tres aspectos: coherencia (el grado de relación entre el ítem y la dimensión en la que se ha incluido), representatividad (se evalúa si el planteamiento del item es el mejor posible para recoger información que precisa) y calidad técnica (mide la redacción del item y si su formulación no induce a ningún tipo de sesgo gramatical). Aquellos que no obtenían una puntuación media de 4 fueron eliminados del cuestionario, excepto si se refería al aspecto de calidad técnica en la que, con una puntuación de 3, fueron admitidos, pero reelaborados en su redacción.

Este artículo se centra en el bloque III, aspectos sociales y familiares, constituido por 28 ítems (la fiabilidad de este bloque es de .762, Tabla 1), que pretenden analizar las situaciones en la familia y en el barrio donde residía el joven en el momento del abandono. En concreto, la influencia de la familia en el momento en que el sujeto decide abandonar sus estudios, tomando como referencia el marco teórico y los estudios empíricos analizados (Albert, 2008; Benito, 2007; Carrillo, Mohamed y Sánchez, 2005; Casquero y Navarro, 2008; CES, 2009; Escardíbul, 2008; Gallardo, 2006; González y López, 2008; Lee y Burkhan, 2000; Marchesi, 2003; MEPSYD, 2008; OECD, 2004; Pajares, 2005, Perkins, 1992; Rontomé y Cantón, 2003; Rué, Amela y Buscarons, 2003; Vaquero, 2005 y Zafra, 2006).

Tabla 1. Contenido de los ítems del bloque III. Aspectos sociales y familiares

\begin{tabular}{ll|l|l}
\hline F62 & $\begin{array}{l}\text { Hábitos de estudios/ Tareas o } \\
\text { deberes }\end{array}$ & F76 & Apoyo emocional familiar \\
\hline F63 & $\begin{array}{l}\text { Hábitos de estudio/Clases par- } \\
\text { ticulares }\end{array}$ & F77 & Expectativas académicas de la familia \\
\hline F64 & Lugar de estudio/biblioteca & F78 & Expectativas laborales de la familia \\
\hline F65 & Apoyo educativo familiar & F79 & Control familiar de las tareas escolares \\
\hline F66 & Lugar de estudio/casa & F80 & Opinión familiar sobre el centro educativo \\
\hline F67 & Lectura en casa & F81 & Control familiar sobre el progreso escolar \\
\hline F68 & Tiempo de estudio & F82 & Confianza de la familia en el joven \\
\hline F69 & Problemas familiares & F83 & Ambiente en el barrio/ drogas \\
\hline F70 & Colaboración familia-escuela & F84 & Ambiente en el barrio/ relaciones sociales \\
\hline F71 & Colaboración familia-escuela & F85 & $\begin{array}{l}\text { Ambiente en el barrio/la escuela como re- } \\
\text { curso }\end{array}$ \\
\hline F72 & $\begin{array}{l}\text { Expectativas de las familias } \\
\text { hacia el estudio }\end{array}$ & F86 & Situación del grupo de amigos \\
\hline
\end{tabular}




\begin{tabular}{cl|l|l}
\hline F73 & $\begin{array}{l}\text { Ayuda de la familia en tareas } \\
\text { educativas }\end{array}$ & F87 & Influencia positiva del grupo de amigos \\
\hline F74 & Control de asistencia a clase & F88 & Participación social religiosa \\
\hline F75 & Ambiente familiar & F89 & Participación social lúdica \\
\hline \multicolumn{2}{c}{ Fiabilidad } & N de casos $=450$ \\
& N de Ítems $=28($ F62 a F89) \\
& Alpha $\alpha=, 7672$ \\
\hline
\end{tabular}

Para detectar una posible falta de sinceridad en las respuestas emitidas a la encuesta, se han elegido los siguientes pares alternos de ítems por su similitud de contenido y se ha analizado la existencia de correlación. La primera pareja está compuesta por los ítems C23 y F62 (Frecuencia con que hacías los deberes en casa y Realizaba las tareas o deberes que me mandaban para casa, respectivamente) siendo su correlación $\mathrm{r}_{\mathrm{C} 23}$, E62 de Spearman $=.672$; p=.000. La segunda, lo forman los ítems E60 y F70 (Frecuencia con que tus profesores hablaban con tus padres y Mis padres acudían al colegio para hablar con los profesorestutores, respectivamente) con una correlación $\mathrm{r}_{\mathrm{E} 60, \mathrm{~F} 70}=.519 ; \mathrm{p}=.000$. Según los resultados obtenidos en este análisis de contingencia inter-ítems, se confirma que las respuestas dadas por los sujetos a estas parejas de ítems, y con las debidas precauciones al resto de la encuesta, son sinceras.

\section{Diseño}

La metodología utilizada es descriptiva, puesto que el objetivo es encontrar respuesta a unos interrogantes cuya explicación científica es aún desconocida. Para ello, se ha utilizado la metodología selectiva de encuesta o surveys (Colás y Buendía, 1998, Cohen y Manion, 2002), dirigida específicamente a jóvenes de entre 16 y 24 años que abandonaron prematuramente los estudios en la ciudad de Melilla. En cuanto al diseño seleccionado en el proceso de recogida de datos se ha optado por uno de tipo transversal, el más utilizado en la investigación por encuesta. Se realizó durante el curso académico 2009-2010, sobre jóvenes que abandonaron los estudios desde el curso 2000-2001 hasta el 2008-2009. 


\section{Procedimiento}

El proceso seguido para la recogida de datos ha pasado por varias fases claramente diferenciadas. En primer lugar, y tras las reuniones oportunas para organizar el trabajo, los investigadores implicados se dirigieron personalmente a los IES para informar a los equipos directivos de la investigación que debíamos llevar a cabo, por encargo del Ministerio de Educación, y solicitarles los datos necesarios. Si bien es cierto que, en un primer momento, muchos se mostraron reacios a nuestros requerimientos porque les suponía un inmenso trabajo en un corto espacio de tiempo, todos lo llevaron a cabo. Dichas bases recogían toda la información que el centro poseía de aquellos alumnos que habían abandonado sus estudios antes de finalizar la etapa obligatoria de la enseñanza secundaria desde el curso 2000-2001.

Se comenzaba de este modo con la segunda fase del estudio: contactar con los sujetos para explicarles el objetivo de la investigación y pedirles su colaboración. Puesto que no se disponía de mucho tiempo, se acordó que el primer contacto debería ser vía telefónica.

La tercera fase empieza cuando se vuelve a contactar con los sujetos, que voluntariamente habían aceptado su participación en la investigación, con el fin de que respondieran al cuestionario que previamente se había elaborado. En este segundo contacto, algunos decidieron abandonar, por lo que finalmente quedó un número de 190 sujetos. Para facilitarles la labor y que no les supusiera una sobrecarga en sus quehaceres diarios, se les ofrecieron varias posibilidades: ir personalmente a la Facultad de Educación y Humanidades y contestarlo en el despacho del grupo de investigación, llevarlo y contestarlo en el momento deseado, o vía telefónica o a través de correo electrónico.

\section{Análisis de Datos}

Para el análisis de los datos obtenidos a través del cuestionario se ha utilizado el paquete estadístico PASW 18.0 (antiguo SPSS) con el fin de dar respuesta al objetivo de la investigación. Se realizó un análisis descriptivo para conocer las creencias de los participantes y un análisis inferencial para estudiar si existían diferencias en función de la procedencia cultural. Para ello se ha utilizado el análisis de varianza para aquellos ítems que utilizan una escala de cinco puntos como forma de respuesta y la prueba chi-cuadrado para los ítems de respuesta dicotómica. 


\section{Resultados}

Para responder al primer objetivo planteado, se estudiaron las variables referentes a la situación socioeconómica de la familia, las circunstancias familiares y el nivel cultural de los progenitores.

El hecho de que los padres se encuentren en situación de desempleo puede ser una dificultad añadida para que estos alumnos tengan la dedicación y concentración suficiente en sus estudios. La cualificación profesional de los padres y su nivel de ingresos determinarán las ayudas económicas que éstos puedan aportar al estudio de sus hijos, lo cual facilitará su permanencia en el sistema educativo y evitará el abandono escolar temprano, que suele estar motivado por la incorporación de estos jóvenes al mercado laboral para aportar recursos económicos a la familia.

Los datos correspondientes a estas circunstancias económicas de la familia son las que aparecen en la siguiente tabla, en la que se detallan los porcentajes obtenidos en la muestra total, especificando la cultura de procedencia.

Tabla 2. Circunstancias económicas de la familia

\begin{tabular}{|c|c|c|c|c|c|c|c|c|}
\hline & \multicolumn{3}{|c|}{ Muestra total } & \multicolumn{2}{|c|}{$\begin{array}{l}\text { Origen euro- } \\
\text { peo }\end{array}$} & \multicolumn{2}{|c|}{ Origen bereber } & \multirow[t]{2}{*}{$\begin{array}{l}\text { Chi cua- } \\
\text { drado }\end{array}$} \\
\hline & Sí & No & $\begin{array}{l}\text { A ve- } \\
\text { ces }\end{array}$ & Sí & No & Sí & No & \\
\hline $\begin{array}{l}\text { ¿Trabajaba } \\
\text { tu padre? }\end{array}$ & $79,4 \%$ & $16,9 \%$ & $3,8 \%$ & $\begin{array}{c}86,6 \\
\%\end{array}$ & $9,8 \%$ & $73,7 \%$ & $23,7 \%$ & $\begin{array}{c}5,59 \\
(p<0,05)\end{array}$ \\
\hline $\begin{array}{l}\text { ¿Trabajaba } \\
\text { tu madre? }\end{array}$ & $27 \%$ & $71,2 \%$ & $1,8 \%$ & $\begin{array}{c}38,8 \\
\%\end{array}$ & $60 \%$ & $14,3 \%$ & $83,1 \%$ & $\begin{array}{c}12,44 \\
(p<0,002)\end{array}$ \\
\hline
\end{tabular}

Existen diferencias significativas, según el grupo cultural, en la ocupación de los padres en el momento de abandonar los estudios; en ambos casos, los padres (chi=5.59; p=.05) y las madres (chi $=12.44 \% ; \mathrm{p}=.002$ ) de la muestra de origen bereber presentan porcentajes más elevados de desempleo que en el caso de los participantes de origen europeo, siendo estas diferencias significativas. En ambas comunidades, las madres presentan porcentajes más ele- 
vados de paro. También encontramos diferencias significativas relacionadas con el nivel de estudios del padre y de la madre (chi $=63.97 ; \mathrm{p}<.000$ y chi $=50.84 ; \mathrm{p}<.000$, respectivamente).

En cuanto a la ocupación principal de los progenitores, se ha podido constatar que el $40 \%$ de los padres eran empleados por cuenta ajena sin cualificación, mientras que, en el caso de las madres, el $45 \%$ se dedicaban al cuidado del hogar. En estas condiciones, los ingresos mensuales aportados por todos los miembros de la unidad familiar que trabajaban fuera de casa, oscilan entre los 1000 y 1499 euros. En este aspecto, los datos confirman que existen diferencias significativas entre los grupos culturales, siendo los ingresos del grupo europeo superiores a los del grupo bereber (chi=43.08; $\mathrm{p}<.000)$. Los ingresos familiares del colectivo europeo son mayoritariamente superiores a 1000 euros, mientras que los del colectivo bereber son inferiores a los 999 euros. Se cuestiona si esta situación económica podía afectar a determinadas condiciones que pudieran repercutir en el momento de estudiar en casa, ya que este aspecto es importante cuando la actividad exige esfuerzo y concentración. También es necesario disponer de tiempo suficiente para ello y tener que simultanearlo con otro tipo de tareas (generalmente domésticas, en el caso de las chicas) o acudir a otro tipo de actividades que limitan el tiempo de dedicación (Tabla 3).

Tabla 3. Condiciones de estudio en casa y su relación en función del grupo cultural

\begin{tabular}{|c|c|c|c|c|c|c|}
\hline Ítem & Nunca & $\begin{array}{l}\text { Algunas } \\
\text { veces }\end{array}$ & $\begin{array}{c}\text { A } \\
\text { menudo }\end{array}$ & Siempre & Medias & $\mathrm{t}$ \\
\hline \multirow{3}{*}{$\begin{array}{l}\text { Tenía un espacio/lugar propio } \\
\text { para estudiar y hacer los debe- } \\
\text { res }\end{array}$} & \multirow{3}{*}{$17,8 \%$} & \multirow{3}{*}{$16,6 \%$} & \multirow{3}{*}{$18,3 \%$} & \multirow{3}{*}{$47,3 \%$} & Europea & \multirow{3}{*}{$\begin{array}{l}2.159 \\
0,032\end{array}$} \\
\hline & & & & & 3,13 & \\
\hline & & & & & $\begin{array}{c}\text { Bereber } \\
2,76\end{array}$ & \\
\hline $\begin{array}{l}\text { Tenía tiempo suficiente para } \\
\text { estudiar }\end{array}$ & $8,9 \%$ & $18,3 \%$ & $32 \%$ & $40,8 \%$ & $\begin{array}{c}\text { Europea } \\
3,28 \\
\text { Bereber } \\
2,81\end{array}$ & $\begin{array}{l}3.532 \\
0.001\end{array}$ \\
\hline $\begin{array}{l}\text { Asistía a algún lugar de ense- } \\
\text { ñanza religiosa (cristiana, mu- } \\
\text { sulmana, hebrea...) }\end{array}$ & $63,9 \%$ & $17,8 \%$ & $8,3 \%$ & $10,1 \%$ & $\begin{array}{c}\text { Europea } \\
1,45 \\
\text { Bereber } \\
1,85\end{array}$ & $\begin{array}{l}-.3 .337 \\
0,002\end{array}$ \\
\hline $\begin{array}{l}\text { Participaba en algún club o } \\
\text { asociación del barrio o la ciu- } \\
\text { dad (deportes, excursiones...) }\end{array}$ & $49,1 \%$ & $23,7 \%$ & $10,1 \%$ & $17,2 \%$ & $\begin{array}{c}\text { Europea } \\
2,09 \\
\text { Bereber } \\
1,79\end{array}$ & $\begin{array}{c}1.533 \\
\text { n.s. }\end{array}$ \\
\hline
\end{tabular}


En la tabla anterior se recoge que el $72.8 \%$ de los entrevistados considera que tenía tiempo para estudiar y sólo el $17.8 \%$ responde que no tenían un lugar adecuado para hacer los deberes. De forma mayoritaria, los encuestados manifiestan que no participaban en actividades lúdicas (deportes, excursiones, etc.), ni asistían a escuelas religiosas $(72.8 \%$ y $81.7 \%$, respectivamente).

En este apartado se observan diferencias significativas debidas a que los participantes de origen bereber presentan medias más altas en la asistencia a lugares de enseñanza religiosa $(\mathrm{t}=-3.337 ; \mathrm{p}=.002)$. Por otra parte, los de origen europeo obtienen valores más altos en los ítems relacionados con tener tiempo suficiente para estudiar $(\mathrm{t}=3.532 ; \mathrm{p}=.001)$ y en tener un espacio propio para el estudio $(\mathrm{t}=2.159 ; \mathrm{p}=.032)$. Los participantes del grupo bereber señalaban, en mayor medida, la asistencia a bibliotecas públicas para realizar actividades académicas (Tabla 3).

Otras circunstancias que pueden influir negativamente en el rendimiento de los alumnos, son los problemas familiares, entre las que destacamos: separación de los padres, violencia doméstica y consumo de sustancias adictivas en el ámbito familiar (Tabla 4). Entre las características relativas a la composición de la unidad familiar se ha podido constatar que, de los que respondieron que vivían con uno solo de los padres (6\%), era porque estaban separados; el $1 \%$ eran hijos de padre/madre soltero/a y el $4 \%$ eran viudos o viudas. Los datos aportados por la muestra encuestada (Tabla 4), ponen de manifiesto que sólo en un porcentaje pequeño se dan estas circunstancias negativas en el seno familiar de los participantes. Únicamente sería destacable el poco tiempo que los padres solían pasar con sus hijos (33.8\% nunca o algunas veces).

Tabla 4. Problemas familiares y la comparación en función del grupo cultural

\begin{tabular}{lcccccc}
\hline Item & Nunca & $\begin{array}{c}\text { Algunas } \\
\text { veces }\end{array}$ & $\begin{array}{c}\text { A } \\
\text { menudo }\end{array}$ & Siempre & Medias & t \\
\hline $\begin{array}{l}\text { Algún miembro de mi familia } \\
\text { tuvo problemas importantes de }\end{array}$ & $69,8 \%$ & $16 \%$ & $4,7 \%$ & $9,5 \%$ & $\frac{1,48}{\text { Bereber }}$ & n.s. \\
$\begin{array}{l}\text { salud, drogas, justicia... } \\
\begin{array}{l}\text { Había discusiones fuertes en } \\
\text { casa }\end{array}\end{array}$ & $54,4 \%$ & $34,4 \%$ & $8,3 \%$ & $5,9 \%$ & $\begin{array}{c}\text { Europea } \\
1,61\end{array}$ & n.s. \\
\hline
\end{tabular}




\begin{tabular}{|c|c|c|c|c|c|c|}
\hline & & & & & $\begin{array}{c}\text { Bereber } \\
1,71\end{array}$ & \\
\hline $\begin{array}{l}\text { Mis padres pasaban su tiempo } \\
\text { libre con mis hermanos y con- } \\
\text { migo }\end{array}$ & $16,6 \%$ & $17,2 \%$ & $29,6 \%$ & $36,7 \%$ & $\begin{array}{c}\text { Europea } \\
3,01 \\
\text { Bereber } \\
2,70\end{array}$ & $\begin{array}{c}\mathrm{t}=2.159 \\
\mathrm{p}=.05\end{array}$ \\
\hline
\end{tabular}

En esta variable no se observan diferencias significativas debidas al grupo cultural de pertenencia (Tabla 4), excepto en el tiempo que los padres dedicaron a sus hijos; en este caso, los europeos manifiestan haber recibido más dedicación de sus padres que los de origen bereber, aproximándose esta diferencia de medias a la significación $(\mathrm{t}=2.159 ; \mathrm{p}=.05)$.

Como última variable a estudiar para la consecución del primer objetivo, se consideró oportuno analizar el nivel de estudios de los padres, ya que el rendimiento de los hijos suele correlacionar con el nivel de estudios que éstos tienen. El estudio reflejó que las madres suelen tener un nivel de estudios inferior al de los padres, aunque sin grandes diferencias. Las mayores frecuencias se alcanzan para un nivel equivalente a Educación Primaria (34.3\% en el caso de los padres y $36.7 \%$ en el caso de las madres). El menor índice de formación se aprecia en aquellos que tienen un nivel inferior al de Educación Primaria (20.7\% en padres y $24.9 \%$ en madres).

Con respecto al nivel de estudios del padre, sí se encuentran diferencias significativas entre los dos grupos culturales participantes ( $\mathrm{chi}=63.97 \%$; $\mathrm{p}<.000$ ), ya que el nivel de estudios de la muestra de origen europeo es superior al del colectivo bereber (el 36\% de los primeros tiene estudios de Educación Secundaria frente al 43.8\% de los segundos que no tiene ninguna titulación). El 31\% de las mujeres europeas tienen estudios de Educación Secundaria frente al $13.8 \%$ de las de origen bereber. Además, el $46.2 \%$ de estas últimas manifiesta que no tiene estudios frente al $5.7 \%$ de las europea.

El segundo objetivo fue conocer la implicación familiar en el progreso educativo de su hijo, para lo que se ha evaluado la actitud que manifiestan los padres hacia varios ítems relacionados con el rendimiento, implicación en el centro educativo, etc.

En lo referente a la preocupación de los padres por los estudios de sus hijos es destacable que el $67,4 \%$ de los encuestados declaran que sus progenitores no asistían nunca al cen- 
tro para hablar con los profesores-tutores o tan sólo iban algunas veces. Sin embargo, el $63.9 \%$ sí hace constar que se preocupaban por sus notas y tan sólo un 5.9\% afirma que la inquietud de la familia por los resultados académicos era inexistente. Estos datos contrastan con la respuesta de que un $34.9 \%$ destaque que sus padres siempre controlaban la realización de los deberes para casa. Hay que resaltar que un $60.9 \%$ señala la nula participación familiar en las actividades del centro educativo, frente a un $20.2 \%$ que contesta que colaboraban a menudo o siempre.

Destacando otros resultados, el $62.1 \%$ dice que siempre que faltaban a clase sus padres se enfadaban y, relacionado con la motivación hacia los estudios un $68 \%$ declara que sus familias siempre les decían que estudiar les ayudaría a encontrar un buen trabajo y que confiaban en su capacidad para tener éxito en los estudios (45.6\%).

Tabla 5. Implicación familiar en el progreso educativo de los hijos y la comparación en función del grupo cultural

\begin{tabular}{|c|c|c|c|c|c|c|}
\hline $\begin{array}{l}\text { Implicación familiar en los es- } \\
\text { tudios }\end{array}$ & Nunca & $\begin{array}{l}\text { Algu- } \\
\text { nas } \\
\text { Veces }\end{array}$ & $\begin{array}{c}\text { A } \\
\text { menudo }\end{array}$ & Siempre & Medias & $\mathbf{t}$ \\
\hline \multirow{2}{*}{$\begin{array}{l}\text { Mis padres acudían al centro } \\
\text { para hablar con los profesores- } \\
\text { tutores }\end{array}$} & \multirow{2}{*}{$19,5 \%$} & \multirow{2}{*}{$47,9 \%$} & \multirow{2}{*}{$14,8 \%$} & \multirow{2}{*}{$17,8 \%$} & $\begin{array}{c}\text { Europea } \\
2,17 \\
\end{array}$ & \multirow{2}{*}{$\begin{array}{l}\mathrm{t}=-3,12 \\
\mathrm{p}=, 05\end{array}$} \\
\hline & & & & & $\begin{array}{c}\text { Bereber } \\
2,46\end{array}$ & \\
\hline $\begin{array}{l}\text { Mis padres participaban en las } \\
\text { actividades del centro }\end{array}$ & $60,9 \%$ & $18,9 \%$ & $9,5 \%$ & $10,7 \%$ & $\begin{array}{c}\text { Europea } \\
1,56 \\
\text { Bereber } \\
1,82\end{array}$ & n.s. \\
\hline $\begin{array}{l}\text { Mis padres se preocupaban por } \\
\text { mis notas }\end{array}$ & $5,9 \%$ & $10,7 \%$ & $18,9 \%$ & $63,9 \%$ & $\begin{array}{c}\text { Europea } \\
3,95 \\
\text { Bereber } \\
3,35\end{array}$ & n.s. \\
\hline $\begin{array}{l}\text { Mis padres o hermanos mayores } \\
\text { me ayudaban a hacer los debe- } \\
\text { res }\end{array}$ & $27,2 \%$ & $29,6 \%$ & $21,3 \%$ & $21,9 \%$ & $\begin{array}{c}\text { Europea } \\
2,39 \\
\text { Bereber } \\
2,34\end{array}$ & n.s \\
\hline $\begin{array}{l}\text { Mis padres se enfadaban cuando } \\
\text { faltaba a clase }\end{array}$ & $11,2 \%$ & $14,8 \%$ & $11,8 \%$ & $62,1 \%$ & $\begin{array}{c}\text { Europea } \\
3,29 \\
\text { Bereber } \\
3,21\end{array}$ & n.s. \\
\hline $\begin{array}{l}\text { Mis padres querían que hiciese } \\
\text { un ciclo formativo o una carrera } \\
\text { que no me gustaba }\end{array}$ & $59,8 \%$ & $11,2 \%$ & $9,5 \%$ & $19,5 \%$ & $\begin{array}{c}\text { Europea } \\
1,99 \\
\text { Bereber } \\
1,79\end{array}$ & n.s. \\
\hline Mis padres creían que estudiar & $5,9 \%$ & $14,2 \%$ & $11,8 \%$ & $68 \%$ & Europea & $\mathrm{t}=1,86$ \\
\hline
\end{tabular}




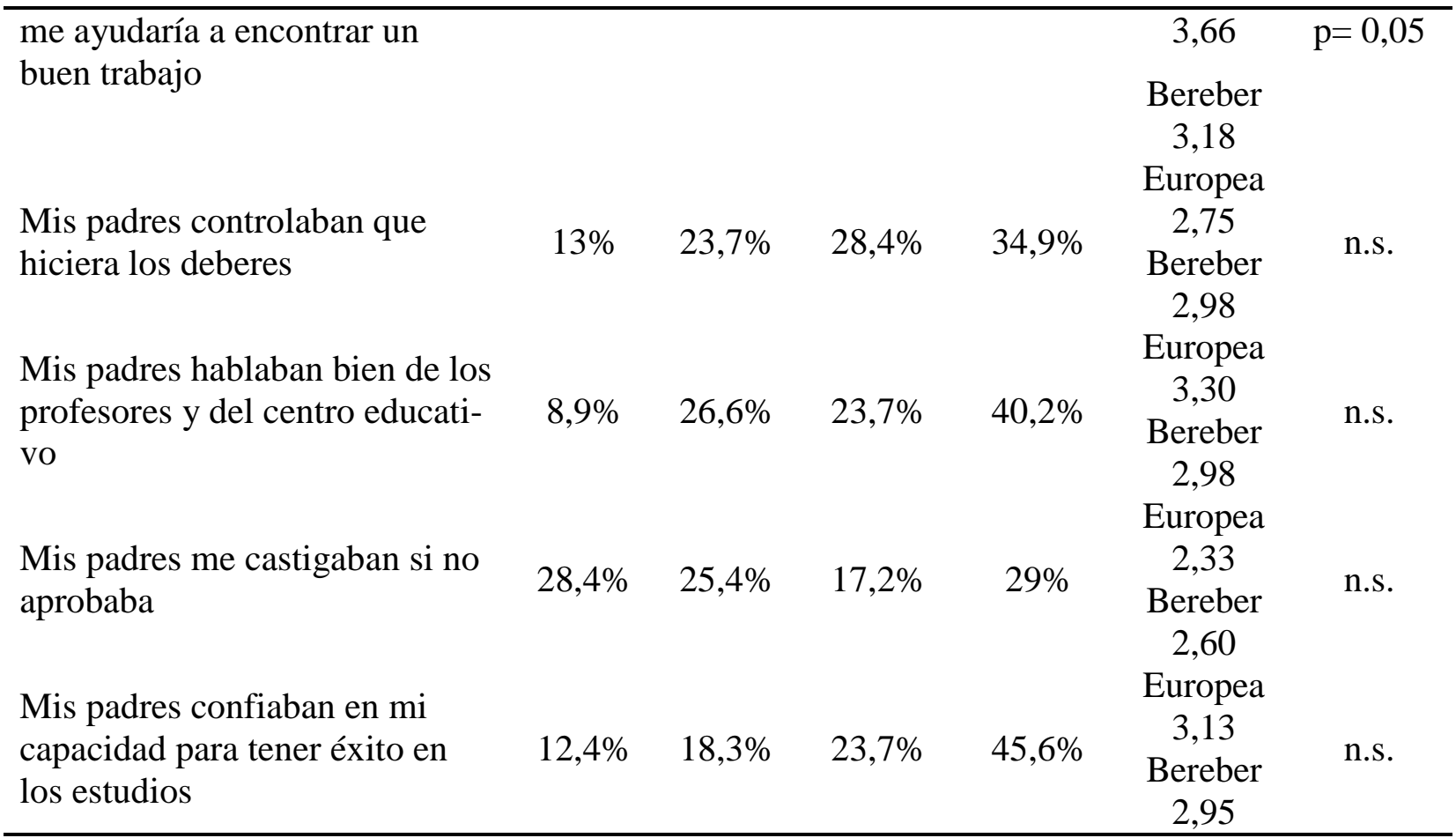

En la tabla anterior se puede observar que no existen diferencias significativas en la actitud de los padres hacia el estudio en función del grupo cultural, salvo en dos ítems; el primero, la frecuencia con la que los padres acudían a hablar con los profesores-tutores $(\mathrm{t}=-, 3,12$; $\mathrm{p}=.05$ ), siendo los participantes de origen bereber los que presentan una media significativamente más alta que la de los europeos. El segundo, la percepción que los padres tienen de la importancia del estudio para encontrar un buen trabajo $(t=1,86 ; p=.05)$, la media es superior en los participantes europeos que en los bereberes.

En cuanto al tercer objetivo, anlizar cómo influye la procedencia cultural en el abandono escolar temprano, los datos indican que hay una proporción similar entre ambos grupos (52.1\% de origen europeo y $47.9 \%$ de origen bereber). En relación con la variable edad, los datos confirman que existen diferencias significativas en la edad media de abandono de los estudios $(\mathrm{t}=2,881 ; \mathrm{p}=, 004)$. Los participantes de origen bereber abandonan con 16.5 años de edad mientras que el grupo europeo presenta una edad media de abandono de casi un año más (17.3). 


\section{Discusión y conclusiones}

Siguiendo a Benito (2007) la importancia del factor familiar viene recogida en la LOGSE, que planteaba la necesidad de establecer y mantener estrechas relaciones con los padres, ya que como la investigación demuestra (Marchesi, 2003; OECD, 2004; Pajares, 2005), el nivel socioeconómico y la preparación de los padres tienen influencia en los resultados académicos de los hijos. De hecho, se ha considerado a la familia como uno de los indicadores para entender el fracaso escolar, tanto en su implicación, como en el nivel socioeconómico o en la dedicación, el apoyo y las expectativas sobre el futuro académico de sus hijos (Marchesi y Pérez, 2003).

Con respecto a los recursos económicos del hogar, se ha encontrado que el nivel de ingresos de la familia de los encuestados oscilaba entre los 1000 y 1499 euros al mes, lo que puede ocasionar que los alumnos necesiten determinadas ayudas al estudio (becas) o algún tipo de incentivo que evite el abandono escolar temprano. Pérez-Díaz, Rodríguez y Sánchez (2001) demuestran que existe una relación directa entre la clase social y la adquisición de recursos como libros de consulta, ordenador, etc.

Los resultados de esta investigación coinciden con los de Lee y Burkhan (2000) que concluyen que a mayor desventaja sociocultural existe mayor riesgo de fracaso. En este sentido, el Informe PISA 2006 señala que el nivel socioeconómico y el nivel de estudios de los padres es un factor influyente, aunque no determinante, en el rendimiento escolar de los hijos.

Según Torío (2004) el contexto familiar debería promover un óptimo desarrollo en los hijos, debido a su influencia educativa como agente de socialización. En este sentido, pueden existir otras circunstancias que influyan negativamente en el rendimiento de los alumnos, como la existencia de problemas familiares (separación de los padres, violencia doméstica, consumo de sustancias adictivas, etc.). Los datos de este trabajo reflejan que sólo en un porcentaje pequeño se dan estas circunstancias negativas en casa de los participantes. Únicamente sería destacable el poco tiempo que pasaban con sus hijos. En relación con el grupo cultural, los de origen europeo manifiestan haber recibido más dedicación de sus padres que los de procedencia bereber, aproximándose esta diferencia de medias a la significación. Siguiendo a Marchesi (2003) las relaciones que los padres establecen con sus hijos, sus códigos comunicativos y lingüísticos, sus expectativas sobre su futuro, el apoyo que les proporcionan en las tareas 
escolares y su participación en las actividades del centro educativo puede hacer que aumente el interés y esfuerzo de su hijo por ampliar sus conocimientos.

En cuanto a la formación de los padres, las personas encuestadas responden que el nivel de estudios de sus progenitores es de Educación Primaria y que los padres tienen una preparación mayor que las madres. Estos resultados están en la línea de Marchesi (2003) que concluye que uno de los factores que pueden condicionar el éxito o fracaso escolar del alumnado es el nivel de estudios de sus padres, siendo más decisivo el de la madre.

En esta investigación las madres tienen una formación inferior a la de los padres, aunque sin grandes diferencias. En esta línea, el informe PISA 2000 (OCDE, 2001) señala que los estudiantes cuyas madres no han terminado los estudios de Educación Secundaria tienen unas puntuaciones en habilidad lectora 44 puntos por debajo de aquellos cuyas madres han finalizado este tipo de estudios. Pérez-Díaz, Rodríguez y Sánchez (2001) han concluido que cuanto mayor es el nivel educativo de los padres, menor es la cantidad de asignaturas suspensas; por esta razón, sólo un 36\% de los estudiantes cuyas familias tienen estudios primarios incompletos logran aprobarlo todo. Sin embargo, un bajo nivel educativo de los padres no siempre desemboca en abandono escolar del hijo. A veces, el bajo nivel de formación puede ser compensado con un mayor compromiso de los progenitores con la educación de sus hijos. Por tanto, "lo importante no es el capital cultural que se posee sino cómo se transmite" (Marchesi, 2003, p.17).

El nivel de estudios de los padres no abarca toda la influencia familiar, otro de los factores que condicionan el éxito o fracaso de los hijos es la profesión que desempeñen los padres y madres, así como el nivel de ingresos que obtenga la unidad familiar (Martínez y Álvarez, 2005). En este sentido, un bajo nivel de formación de los progenitores incide en el desempeño profesional. En esta investigación, el $40 \%$ de los padres de los participantes eran empleados por cuenta ajena sin cualificación mientras que, en el caso de las madres, el $45 \%$ se dedicaba al cuidado del hogar.

Además, la familia ha de fomentar una actitud positiva hacia el estudio (Marjoribanks, 2003), ya que este entorno influye en el éxito escolar. Aunque los datos del Informe PISA muestran que un rendimiento pobre en la escuela no es consecuencia automática de un entorno socioeconómico desfavorecido, el ambiente familiar parece influir poderosamente en el 
rendimiento (Belinchón, Calero, De la Encina, González, Herrero, Martínez, Olabuenaga, y Tesa, 2009).

Según la implicación de los padres hacia el centro educativo y los estudios de los hijos, se destaca que los encuestados responden que sus progenitores no asistían nunca al centro para hablar con los profesores-tutores, aunque la mayoría contesta que sólo iba algunas veces. Sin embargo, un alto porcentaje de alumnos considera que se preocupaban por sus notas y tan sólo un 5.9\% responde que el interés por los resultados académicos era inexistente. También se resalta que un $60.9 \%$ señala la nula participación de la familia en las actividades del centro educativo. En esta línea se encuentran los resultados de Meil (2006) que considera que al menos un $14 \%$ de los padres y madres hacen dejación de sus responsabilidades como educadores. Esta falta de compromiso es mayor en padres con bajos niveles culturales aunque, lamentablemente, se da en todo tipo de familias. De esta forma, cuando los padres y madres participan activamente en el centro escolar, los hijos incrementan su rendimiento académico y el centro mejora su calidad educativa (Marchesi y Martín, 1998). En esta línea, en un estudio realizado por Gil (1994), se establece como indicador de la implicación de las familias el número de veces que los padres acuden al colegio o al instituto. Este autor concluye que existe escasa participación de los padres en general y que ésta se limita a aspectos credencialistas (casi exclusivamente interés en la marcha académica de los hijos). López y Encabo (2001) subrayan la importancia de la colaboración familiar e instituciones educativas para conseguir reducir el distanciamiento en cuanto a objetivos educativos. Cuanto menor sea el nivel educativo y cultural de los padres más se limitan sus estrategias de acción en el hogar y mayor el distanciamiento con el centro educativo, por lo que la influencia educadora de la familia se resiente (Marchesi, 2003).

En relación con el grupo cultural de pertenencia, se encuentran diferencias significativas acordes con el interés de los padres por los estudios de sus hijos en dos ítems, el primero sobre la frecuencia con la que éstos acudían a hablar con los profesores-tutores, siendo los participantes del grupo bereber los que presentan una media significativamente más alta que los de procedencia europea. El segundo ítem, la percepción que los padres poseen de la importancia del estudio para encontrar un buen trabajo, presenta un porcentaje mayor entre los participantes de origen europeo que entre los participantes bereberes. Oliva y Palacios (2003) señalan que los padres que participan más en la vida del centro escolar y tienen más interés en lo relacionado con la educación de sus hijos tienen hijos con mayor nivel lingüístico, mayor 
rendimiento y menos problemas de conducta y, además, participando en el centro transmiten actitudes favorables al mismo.

Podemos concluir que el perfil socio-familiar del alumno con abandono escolar temprano es el siguiente:

1. El nivel de estudios de los padres y madres de los participantes de procedencia europea es superior a los padres y madres del grupo de origen bereber que, en mayor porcentaje, no posee ninguna titulación.

2. Los mayores porcentajes de desempleo se observan en el grupo de familias de origen bereber, siendo las madres las que presentan mayor tasa de desempleo.

3. En cuanto al nivel de ingresos de la unidad familiar, es el grupo de procedencia europea el que obtiene ingresos superiores a 1000 euros, frente a una cifra inferior a los 999 euros del grupo de origen bereber.

4. La actitud de los padres hacia el estudio es similar en ambos grupos, las diferencias se centran en el item la frecuencia con la que los padres acudían a hablar con los profesores-tutores y la percepción que tienen los padres de la importancia del estudio para conseguir un buen trabajo. En el primer ítem, son los padres de los participantes de origen bereber los que presentan una media significativamente más alta que los de origen europeo. Por el contrario, en el segundo ítem, los participantes de origen europeo obtienen mayores puntuaciones.

5. Los participantes de origen europeo son los que manifiestan que tienen un espacio adecuado y tiempo suficiente para estudiar. Por el contrario, los participantes de procedencia bereber manifiestan que asisten más a la biblioteca para hacer los trabajos y estudiar.

6. Aunque no existen diferencias significativas, es el grupo de participantes de origen bereber el que presenta medias significativamente más altas que los de origen europeo en la asistencia a lugares de enseñanza religiosa.

7. Los participantes de procedencia europea consideran que sus padres les dedicaron más tiempo que los de procedencia bereber.

Por todo esto, es importante que los profesionales de la educación faciliten cauces para que las familias se impliquen más en la educación de sus hijos, a través de la creación de Escuelas de Padres que oferten programas formativos adaptados a las características de las fami- 
lias del alumnado matriculado en los distintos centros, poniendo un énfasis especial en la colaboración de aquéllas que se encuentren en situación de desventaja sociocultural.

Para ayudar a las familias en el apoyo a los alumnos, se necesita que éstos se sientan identificados con su centro educativo, convirtiéndose en una alternativa importante para ocupar el tiempo libre. Para conseguir este propósito, los centros han de ofertar un programa de actividades extraescolares interesante, abierta al barrio y de calidad, en colaboración con la administración educativa, organizaciones no gubernamentales o con diferentes convenios con instituciones locales y nacionales (ME, 2010).

\section{Referencias}

Albert, C. (2008). Exclusión social y pobreza: transición educativo-formativa e inserción laboral de la población joven. Madrid: Ministerio de Trabajo e Inmigración (Informe FRIPOS).

Belinchón, J., Calero, J., De la Encina, P., González, P., Herrero, C., Herrero, R., Martínez, J.C., Olabuenaga, A. y Tesa, M.V. (2009). Fortalecer los compromisos entre familia y escuela. Un ejemplo de buena práctica. Comunidad de Madrid: Consejo Escolar.

Benito, A. (2007). La LOE ante el fracaso, la repetición y el abandono escolar. Revista Iberoamericana de Educación, 43 (7), 1-11.

Buendía, L. (1997). La investigación por encuesta. La investigación observacional. En L. Buendía, P. Colás y F. Hernández (Coords.). Métodos de investigación en psicopedagogía. Madrid: McGraw Hill.

Calero, J., Choi, A. y Waisgrais, S. (2010). Determinante del riesgo de fracaso escolar en España: una aproximación a través de un análisis multinivel aplicado a PISA 2006. $R e$ vista de Educación, Número Extraordinario 2010, 225-256.

Carrillo, P., Mohamed, I. y Sánchez, L. (2005). Actuaciones del Departamento de Orientación en el absentismo escolar. En M.A. Gallardo (Coord.). Evaluación e intervención en contextos educativos. Melilla: Facultad de Educación y Humanidades.

Casquero, A. y Navarro, Ma . L. (2008). El abandono escolar temprano en el periodo LOGSE. En I. Neira, N. Porto, N. Lamelas, A. Filgueira, A. Vaquero, S. Fernández, E. Vázquez, y E. Vieira (Coords.). Investigaciones en economía de la educación, 3, 173181. Santiago de Compostela: AEDE.

CES (2009). Informe 01/2009: Sistema educativo y capital humano. Madrid: Departamento de Publicaciones del Consejo Económico y Social. 
Cohen, L. y Manion, L. (2002). Métodos de investigación educativa. Madrid: La Muralla.

Colás, P. y Buendía, L. (1998). Investigación educativa (3ª ed.). Sevilla: Alfar.

Commision of the European Communitie (2011). Progress towards the Common European objectives in education and training. Indicators and benchmarks 2011. Commision of the European Communitie. Bruselas.

Escárdibul, J.O. (2008). Los determinants del rendimiento educativo en España. Un análisis a partir de la evaluación PISA-2006. En I. Neira, N. Porto, N. Lamelas, A. Filgueira, A. Vaquero, S. Fernández, E. Vázquez, y E. Vieira (Coords.). Investigaciones en Economía de la Educación, 3, 173-181. Santiago de Compostela: AEDE.

Feito, R. (2009). Éxito escolar para todos. Revista Iberoamericana de Educación, no 50,131 151.

Fernández Enguita, M., Mena Martínez, L. y Riviere Gómez, J. (2010). Fracaso y Abandono Escolar en España. Colección Estudios Sociales, 29. Fundación La Caixa.

Finn, J.D. (1993). School engagement y students at risk. Washington, DC. National Center for Educational Statistics, U.S: Department of Education.

Gallardo, L. (2006). "Sobresaliente” para España en abandono escolar. En A. Mas. Aprende más, 1-2. Disponible en http://www.aprendemas.com/reportajes/pdf/plantillaa.pdf

Gil, F (1994). Investigando las relaciones familia-escuela. La participación de los padres en el centro. Revista Aula. Vol. VI, 67-76.

González, Mª T. (2006). Absentismo y abandono escolar: una situación singular de la exclusión educativa. Revista Iberoamericana sobre Calidad, Eficacia y Cambio en Educación, 4 (1), pp. 1-14. Disponible en http://www.rinace.net/arts/vol4num1/art1_htm.htm

González, S.M. y López Puig, A. J. (2008). Evolución del resultado educativo en Espala según PISA 2003 y 2006. En I. Neira, N. Porto, N. Lamelas, A. Filgueira, A. Vaquero, S. Fernández, E. Vázquez, y E. Vieira (Coords.). Investigaciones en economía de la educación, 3, 173-181. Santiago de Compostela: AEDE.

Instituto Vasco de Evaluación e Investigación Educativa (IVEI) (2007). Abandono escolar. Segundo ciclo de la ESO. San Sebastián: Gobierno Vasco.

Lee, V.E. y Burkhan, D. T. (2000). Droping Out of High School: the Role of School Organization and Structure. Disponible en:

http:// www.civilrightproject.harvard.edu/research/dropoust/lee.pdf

Lehr, C.A., Johnson, D. R. Bremer, Ch. D. Cosio, A. y Thompson, M. (2004). Essential Tools. Increasing rates of school completion: moving fron policy and research to 
parctice. A manual for policymakers, administrators and educators. Disponible en http://www.nceset.org/publications/essencialtools/dropout.pdf

López Rupérez, F. (2010). La dimensión del problema. El fracaso escolar en España desde una perspectiva autonómica e internacional. En A. Canalda y otros (2010), En busca del éxito educativo: realidades y soluciones, 27-52. Madrid: Fundación Antena 3.

López, A. y Encabo, E (2001). Mejorar la comunicación en niños y adolescentes. Madrid: Pirámide.

Lozano, A. (2003). Factores personales, familiares y académicos que afectan al fracaso escolar en la Educación Secundaria. Electronic Journal of Research in Educational Psychology, 1 (1), 43-66.

Marchesi, A. (2003). El fracaso escolar en España. Madrid: Fundación Alternativa. Disponible en http://www.falternativas.org/base/download/024_29-07-05_11_2003.pdf

Marchesi, A. y Martín, E. (1998). Calidad de la enseñanza en tiempo de cambio. Madrid: Alianza.

Marchesi, A. y Pérez, E. (2003). La comprensión del fracaso escolar en España. En A. Marchesi y C. Hernández Gil (Coords.). El fracaso escolar. Madrid: Alianza.

Marjoribanks, K. (2003). Family background, individual and environmental influences, aspirations and young adults'educational attainment: a follow up study. Educational Studies, 29 (2-3), 233-242.

Martínez, R.A. y Álvarez, L. (2005). Fracaso y abandono escolar en Educación Secundaria Obligatoria. Aula Abierta, 85, 127-146.

Meil, G. (2006). Padres e hijos en la España actual. Colección Estudios Sociales. Fundación la Caixa. Barcelona. Disponible en:

http://www.fundacion.lacaixa.es/estudiossociales/vol119_eshtmil

Ministerio de Educación (2010). El abandono escolar temprano en las ciudades de Ceuta y Melilla. Madrid: Secretaría General Técnica.

Ministerio de Educación, Política Social y Deporte (MEPSYD) (2008). Plan para la reducción del abandono escolar. Documento de trabajo. Madrid: MEPSYD.

Newman, F.M., Wehlage, G. y Lamborno, S.D. (1992). The significance and sources of student engagement. En F.M. Newman (Ed.). Student engagement and achievement in American Secondary Schools. New York: Teacher Colleges Press.

OCDE (2001). La medida de los conocimientos y destrezas de los alumnos. La evaluación de la lectura, las matemáticas y las ciencias en el Proyecto Pisa 2000. Madrid: MECD. 
OECD (2004). Learning for tomorrow's first results from PISA 2003. Paris. OECD publications. Disponible en http://www.oecd.org/dataoecd/42/54/43638848.pdf

OECD (2009). Panorama de la educación 2009: indicadores de la OCDE. Resumen en español de Education at a Glance 2009: OECD indicators. Paris. OECD publications. Disponible en http://www.oecd.org/dataoecd/42/54/43638848.pdf

Oliva, A. y Palacios, J. (2003). Familia y escuela: padres y profesores. En M.J. Rodrigo y J. Palacios (coord.). Familia y desarrollo humano (333-349). Madrid: Alianza Editorial.

Pajares, R. (2005). Resultados en España del estudio PISA 2000: conocimientos y destrezas de los alumnos de 15 años. Madrid. INECSE. Disponible en http://www.ince.mec.es/pub/pisa2000infnacional.pdf.

Pérez-Díaz, V., Rodríguez, J.C. y Sánchez, L. (2001). La familia española ante la educación de sus hijos. Barcelona: Fundación la Caixa.

Perkins, D.N. (1992). Smart schools: from training memories to educating minds. New York: Free Press.

Poy, R. (2010). Efectos del credencialismo y las expectativas sociales sobre el abandono escolar. Revista de Educación, Número Extraordinario 2010, 147-169.

Railsback, J. (2004). Increasing student attendance: strategies from research and practice. Disponible en http://www.nwrel.org/request/2004june/textonly.html

Rontomé, C. y Cantón, J.M. (2008). Estudio sociológico sobre la juventud en Ceuta. Un análisis cualitativo. Ceuta: Sociópolis. Estudios Sociológicos. (Estudio inédito).

Robledo, M., Cortez, J. y Cortez, A. (2004). Droppout Prevention Programs. Right intent, wrong focus and some suggestions on where to go from here. Education and Urban Society, 36 (2), 169-188.

Roca, E. (2010). El abandono temprano de la educación y la formación en España. Revista de Educación, Número Extraordinario 2010, 31-62.

Rubenger, R.W. (2001). Why students drop out of school and what can be done. Disponible en http://www.civilrightsproject.harvard.edu/reseacrh/dropousts/rebenger.pdf

Rué, J., Amela, M. y Buscarons, M. (2003). El absentismo escolar como reto para la calidad educativa. Premios Nacionales de Investigación e Innovación Educativa (Recopilación de resúmenes de los trabajos premiados por el ME), 2, 103-125.

Segura Vázquez, P. (2010). Pobreza y exclusión social. Diagnóstico de los distritos 4 y 5 de Melilla. Informe inédito encargado por el Ministerio de Trabajo e Inmigración, el INEM y Acción Social sin Fronteras.

Torío, S. (2004). Familia, escuela y sociedad. Aula Abierta, 83, 35-52. 
Vaquero, A. (2005). El abandono escolar temprano en España: programas y acciones para su reducción. Revista Galega o Ensino, 47, 1443-1464.

Wilms, J.D. (2000). Student engagement and school. A sense of belonging and participation. Results from PISA 2000. OECD. Disponible en:

http://www.pisa.oecd.org/Docs/download/StudentEngagement.pdf

Zafra, M. (2006). La formación del profesorado para la diversidad social. Revista de Educación, 339, 147-168. 